\title{
The peculiar electrical response of liquid crystal-carbon nanotube systems as seen by impedance spectroscopy
}

\author{
A García-García , R Vergaz , J F Algorri , M A Geday and J M Otón
}

\begin{abstract}
Conductive nanoparticles, especially elongated ones such as carbon nanotubes, dramatically modify the electrical behavior of liquid crystal cells. These nanoparticles are known to reorient with liquid crystals in electric fields, causing significant variations of conductivity at minute concentrations of tens or hundreds ppm. The above notwithstanding, impedance spectroscopy of doped cells in the frequency range customarily employed by liquid crystal devices, 100 $\mathrm{Hz}-10 \mathrm{kHz}$, shows a relatively simple resistor/capacitor response where the components of the cell can be univocally assigned to single components of the electrical equivalent circuit. However, widening the frequency range up to $1 \mathrm{MHz}$ or beyond reveals a complex behavior that cannot be explained with the same simple EEC. Moreover, the system impedance varies with the application of electric fields, their effect remaining after removing the field. Carbon nanotubes are reoriented together with liquid crystal reorientation when applying voltage, but barely reoriented back upon liquid crystal relaxation once the voltage is removed. Results demonstrate a remarkable variation in the impedance of the dielectric blend formed by liquid crystal and carbon nanotubes, the irreversible orientation of the carbon nanotubes and possible permanent contacts between electrodes.
\end{abstract}

Keywords: impedance spectroscopy, carbon nanotube, liquid crystal, electric equivalent circuit, electrical response

(Some figures may appear in colour only in the online journal)

\section{Introduction}

In recent years, nanoparticles (NPs) dispersions in liquid crystal (LC) matrices have gained increasing research interest $[1,2]$. The mixture may show modifications in the elastic and dielectric LC constants [3-5], the electro-optical properties $[6,7]$ or the cell conductivity $[3,6,8,9]$.

Liquid crystals are self-organized anisotropic fluids that exhibit long-range orientational order in molecular aggregations called domains, in which molecules align approximately about a preferred orientation called director. If confined into thin (several $\mu \mathrm{m})$ cells and the internal cell walls are adequately conditioned (surface anchoring) single domains may be obtained, as customarily happens in LC displays. As LCs are fluid and feature electric and magnetic anisotropy, any induced orientation may be modified by application of external electric or magnetic fields above a certain threshold. The reorientation occurs to minimize the system energy derived from the surface anchoring elastic forces and the applied field.

LCs, made of elongated molecules, have been used to host elongated dopants such as carbon nanotubes (CNTs) [10]. Coupling between host and dopant may impose an orientation of the latter following the orientational order of the liquid 
crystal. LC reorientation governed by the action of external electric or magnetic fields has been reported to reorient the dopant CNT. Moreover, it has been demonstrated [11] that the order induced in the CNTs is maintained upon removal of the LC.

The effects of elongated nanodopants on LC cell properties have been studied for many years, since the pioneering works of de Gennes [12] on ferronematics and magnetic particles. The classical theoretical study of Burylov and Raikher [13] on anisometric particles explored in depth the coalignment of the LC and the nanoparticle in conditions of rigid (strong) anchoring and weak anchoring. This point is crucial to predict the behavior of LC-nanoparticle blends. Coupling of the LC director field to the nanoparticle surface is determined [14] by the so-called penetration length $\xi$ :

$$
\xi=\frac{K}{W}
$$

where $K$ is the corresponding Frank elastic constant (or an average of them) and $W$ is the polar or azimuthal anchoring energy of the LC molecule to the nanoparticle surface. The penetration length is a measure of the distance where the perturbation caused by the introduction of the nanoparticle induces deformations in the previous nematic order [14]. The most relevant conclusion is that the weak anchoring limit leads to negligible deformations while the rigid anchoring may induce topological defects.

Carbon nanotubes are particularly interesting within this topic, since their reorientation with external fields [15-18] arises the possibility of preparing simple devices whose electrical conductivity can be externally controlled [7, 17-19]. CNTs are anisotropic NPs formed by one or several rolledup graphene sheets. Depending on the rolling direction and the diameter, single-walled carbon nanotubes (SWCNTs) are metallic or semiconductive while multi-walled carbon nanotubes (MWCNTs) are always metallic. The electric conductivity is higher along the CNT longitudinal axis than in the transversal direction [20]. Longitudinal electronic transport is ballistic (i.e. without scattering), enabling the CNT to carry high currents with essentially no heating [21].

According to the anchoring considerations mentioned above, CNTs - either SWCNTs or MWCNTs - are usually on the weak anchoring limit, since their diameters $D \ll \xi$. This means that CNT doping of LCs should not affect the original order of the LC material, as long as the CNT concentration is kept low enough to avoid interactions between CNTs themselves. It is important to note, however, that the coupling between CNTs and LC molecules is so strong that in practice the CNT induces the alignment of at least a thin layer of LC material, which remains bound to the CNT when electric or magnetic fields are applied, or temperature is modified [14].

Any dopant above a certain concentration produces modifications on the host properties. CNTs in LC cells are known to modify fundamental parameters such as response time, black flow and image sticking [10], all of which have direct impact in the LC device manufacturing. Controlling CNT alignment in the LC opens up a way to optimize these parameters. The control should be based on the interactions

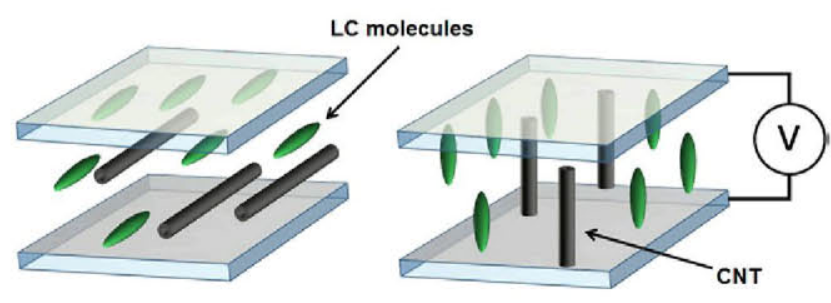

Figure 1. Sketch of LC reorientation with transversal electric field. The CNT contribution to sample conductivity is largely enhanced upon reorientation.

between CNTs and LC molecules (stacking energy or alignment strength) what result crucial in the CNT alignment [4]. Nevertheless, the LC role in the CNT alignment mechanism is not fully understood [22].

The LC induced alignment and reorientation properties may regulate the orientation of dispersed MWCNTs (figure 1). As CNTs are conductive, the possibility of establishing electrical connections between the electrodes of the LC cells arises; these connections could be driven by the applied external field. Furthermore, the presence of CNTs in the material and their reorientation modify the characteristic impedance of the LC. The description of the electrical behavior of CNT-doped LC cells under different conditions could open the possibility to simulate or design internal operations of these systems.

The usual way to describe the electric performance of these elements is to match them with an electric equivalent circuit (EEC). Although EECs of different types and configurations of LC cells have been reported [23-25], a description of the electrical behavior of CNT-doped LC cells in a wide range of frequencies and voltages has not been performed yet. Once the cell behavior has been mirrored to elementary electrical components (resistors, capacitors, coils, etc), every one standing for a physical effect, the contribution of each cell component-LC, CNTs, external circuitry- to the electrical parameters may be established.

This work compares the evolution of EEC components required to describe MWCNT-doped and undoped LC cells, as a function of the frequency and the driving voltage. The evolution of this EEC directly depends on the variations of electrical permittivity induced by LC-and, presumably, MWCNT-reorientation.

\section{Description of the system}

Let us consider a typical LC cell formed by two parallel plates with conductive inner surfaces, located at a few $\mu \mathrm{m}$ to each other. The LC is sandwiched between the plates, and a preferred molecular orientation is induced by previous surface conditioning. Nematic LC materials are customarily made of organic elongated molecules that lie nearly parallel to each other determining an averaged director; besides this orientational order, no relation between the molecular positions exist. We shall assume that the alignment is homogeneous, i.e. the director is parallel to the plates. Moreover, the dielectric anisotropy of the LC material shall be positive, i.e. the dielectric permittivity along the molecular long axis is higher than 
the dielectric permittivity in the perpendicular axis over the whole frequency range under study.

LC cells were prepared using two $0.7 \mathrm{~mm}$ thick ITO-coated glass plates $(100 \Omega / \square)$ from Glasstone, separated $8.25 \mu \mathrm{m}$; the active area is $1 \mathrm{~cm}^{2}$. Homogeneous alignment was induced using a solution of PEDOT : PSS [poly (3, 4-ethylenedioxythiophene) poly (styrenesulfonate)] in water $(1.3 \% \mathrm{w} / \mathrm{w})$. The solution was spin-coated at $4500 \mathrm{rpm}$ and mechanically buffed. Further details on the manufacturing process have been reported elsewhere [9].

It must be mentioned that other polymers like polyimide or polyvinyl alcohol (PVA) are customarily employed as alignment layers for these LC devices, since they induce more efficient aligning surfaces. However, PEDOT : PSS has been preferred in this case since its conductivity is remarkably higher than polyimide or PVA conductivity. This is essential to keep electrical continuity across the system.

Under these conditions, if a voltage above a certain threshold - the Freedericksz transition voltage $\left(V_{\text {th }}\right)$ - is applied between the plates, the LC molecules reorient towards a perpendicular direction, since the electric energy of the system is minimized when the higher dielectric constant lies parallel to the electric field. Increasing further the voltage, the effect saturates and the director adopts a perpendicular, or homeotropic, orientation.

The LC chosen for this work is the positive nematic mixture MLC-6290-000 (Merck). The LC features an optical birefringence $(\Delta n)$ of 0.120 at $588 \mathrm{~nm}$ and a relative dielectric anisotropy $(\Delta \varepsilon)$ at low frequencies of about 10 .

If the LC is doped with MWCNTs, it is expected that these nanoparticles modify their orientation as well. If the cell thickness is similar to the conductive NP length, the MWCNTs should generate a noticeable variation of the impedance and possibly, the electrical connection between the inner plate electrodes. MWCNTs (Sigma Aldrich) employed in this study have an outer diameter of $6-9 \mathrm{~nm}$ and a length of about $5 \mu \mathrm{m}$. The LC-MWCNT suspension was prepared by ultrasonication stirring of $0.01 \% \mathrm{w} / \mathrm{w}$ of MWCNTs in nematic phase. Heating the suspension above the LC to isotropic liquid transition temperature leads to agglomeration of the CNTs.

\section{Electric equivalent circuit}

Impedance Spectroscopy is a powerful method to investigate the dynamics of bound or mobile charges in the bulk or interfacial regions of any solid or liquid material [26]. In this case, the characteristic impedance is studied to develop an EEC describing its behavior. Each component of this circuit is linked to a different physical parameter of the samples. The variation of these components with the driving voltage provides information about changes in the inner structure of the sample (i.e. reorientation of LC molecules and MWCNTs) and other physical phenomena.

Several EECs have been proposed to describe the electrical behavior of LC devices [23-25]. A simple EEC has been recently demonstrated [9] to justify the behavior of MWCNT/ LC composites at intermediate frequencies; yet when the

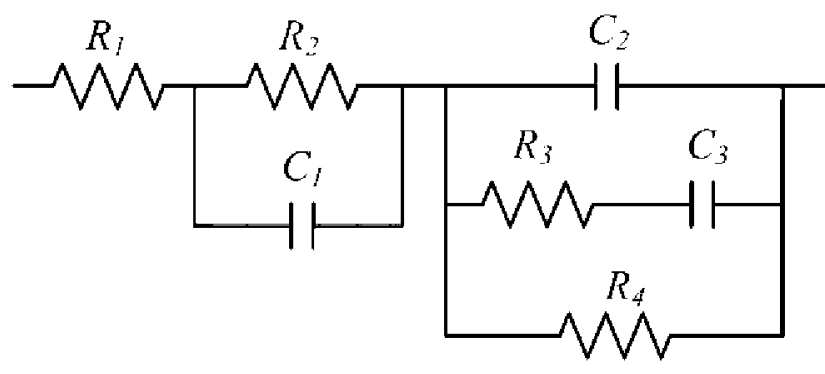

Figure 2. EEC proposed to model the MWCNT-LC composite cells.

frequency range is widen up, a more involved EEC model is required.

The most common EEC used to describe nematic LC cells [23] is shown in figure 2. To our knowledge, this has only been applied to undoped LC cells; our purpose is to check whether MWCNT-doped cells can be modeled as well, either with the same EEC or modifying some components as a function of the frequency and applied voltage.

Each component has a physical significance that describes the different elements contributing to the sample response:

- R1 resistor represents the resistivity coming from connectors and electrodes; due to its physical significance, it must be present in every driving voltage case of study.

- C1 is a double layer capacitor formed in the interface of the alignment layers with the dielectric.

- $\mathrm{R} 2$ resistor is related with the conductivity of the substrate layers.

- C2, C3 and R3 form the circuit employed to describe the Debye dielectric relaxation [26]. C2 results from electronic and atomic polarization. These processes are detected at high frequencies. C3 describes the slow orientation of dipolar polarization which is detected at frequencies lower than $\mathrm{C} 2$ processes. R3 represents the dipolar displacement (heat dissipation).

- R4 represents the conductivity of the dielectric blend (LC with or without MWCNTs).

\section{Characterization method: driving waveform}

Impedance spectroscopy customarily employs sufficiently small voltage signals $\left(100 \mathrm{mV}_{\text {rms }}\right)$ for the system response to be linear. If the study includes the effect of external electric fields, as in this case, the ac signal probe should be superimposed to a bias (offset) dc voltage. However dc voltage leads to electrolytic degeneration of the LC cell by ion generation and migration, and eventual adsorption of the charges onto the alignment layers. To avoid this issue, dc bias has been substituted by a low frequency $(1 \mathrm{~Hz})$ ac square wave to which the low amplitude ac probe voltage signal is added up (figure 3 ). Being a square signal, reorientation of the LC is minimally affected by polarity changes; yet sampling is performed near the end of every cycle to allow the LC to stabilize further. Data from positive and negative half-cycles were separately collected to check for deviations from each other. No significant deviations were found. 


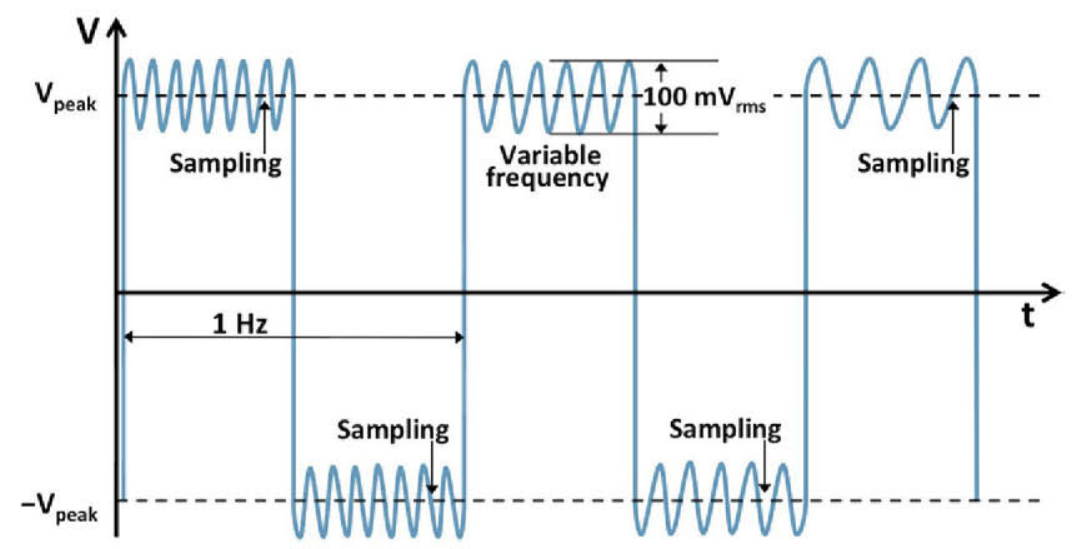

Figure 3. Driving waveform for impedance measurements. Peak voltage ( $\left.V_{\text {peak }}\right)$ and $V_{\text {rms }}$ of the square signal coincides with each other. A variable-frequency sine wave of $100 \mathrm{mV}_{\text {rms }}$ is superimposed. Sampling is done at the end of each half-cycle.

The EEC model has been tested in a frequency range varying from $1 \mathrm{MHz}$ down to $100 \mathrm{~Hz}$. The presence of ions in conventional LC cells is responsible for the behavior below $100 \mathrm{~Hz}$, essentially a non-Debye relaxation process [27] due to surface effects; the ionic relaxation processes follow the electric field at such a low frequency, effectively contributing to the dielectric permittivity. On the other hand, the contribution of cables and contacts complicates the dielectric response above $1 \mathrm{MHz}$; new elements would be required for the EEC to fit the experimental response in the high frequency limit. In practice, $1 \mathrm{MHz}$ seems to be a reasonable high limit for LC structures, since their response and relaxation times are usually in the ms range [28].

Three different square voltage amplitudes, $V_{\text {peak }}=0 \mathrm{~V}_{\mathrm{p}}$, $3 \mathrm{~V}_{\mathrm{p}}, 8 \mathrm{~V}_{\mathrm{p}}$, have been used. In our sample configuration, these correspond to voltage levels below the Fréedericksz threshold, intermediate, and close to saturation respectively. A sinusoidal signal of $100 \mathrm{mV}_{\text {rms }}$ was added up in all cases (figure 3). The waveform was programmed in a 1260 Solartron impedance analyzer controlled by a PC that performs data collection as well. The EEC components are obtained from the EEC model by fitting the experimental results and the parameters through a non-linear least-squares Levenberg-Marquardt algorithm employing the software package ZView (Scribner) version 3.3f.

\section{Results and discussion}

\section{Bode plots}

The impedance measurements were carried out following a driving sequence to monitor the variation and the reversible character of the samples. This sequence is: $0 \mathrm{~V}_{\mathrm{p}}$ (planar alignment), $3 \mathrm{~V}_{\mathrm{p}}$ (an intermediate state between the threshold and saturated voltages), a second measurement at $0 \mathrm{~V}_{\mathrm{p}}, 8 \mathrm{~V}_{\mathrm{p}}$ (saturated voltage) and a new scan at $0 \mathrm{~V}_{\mathrm{p}}$. Switching is considered reversible if all results at all $0 \mathrm{~V}_{\mathrm{p}}$ are coincident. Reversibility might then depend on the time elapsed between the different steps of the driving sequence; as seen below, this has not been the case in any sample batch.

The impedance measurements are shown in Bode plots (figures 4 and 5). The impedance magnitude and phase measurements are represented in separated plots for each sample case (i.e. undoped and doped). The symbols indicate the measurements and the superimposed lines are the EEC fittings.

The impedance measurement of undoped LC cells (figure 4) presents a capacitor effect dominating over a wide band of frequencies for all polarization voltages. In an ideal capacitor, the impedance magnitude should be a straight line (loglog scale) tilted downwards for increasing frequencies while the phase should be a constant horizontal line at $-90^{\circ}$. The LC reorientation increases the dielectric permittivity when increasing the bias voltage, due to the orientation of their axis with the higher dielectric permittivity along the electric field. Consequently, the capacity increases and the impedance magnitude decreases, maintaining the capacitive character in the whole frequency range. The device at these frequencies behaves electrically simply as a capacitor with a dielectric permittivity variable with $V$. As expected, the behavior is fully reversible, as seen by the overlapping of all $0 \mathrm{~V}_{\mathrm{p}}$ measurements. Time elapsed between measurements is not relevant for the results.

Results of impedance measurements of MWCNT-doped LC cells (figure 5) differ significantly from the previous behavior, which reveals an internal change in the dielectric character of the previous capacitor. The first $0 \mathrm{~V}_{\mathrm{p}}$ run shows a remarkable (3.5 $\times$ ) decrease of both the magnitude and phase of impedance at low frequencies. Therefore, the presence of MWCNTs in the dielectric blend modifies the electric behavior of the unswitched LC sample. The dominating capacitor behavior is shown by the impedance magnitude from low to high frequencies, yet the phase matches the undoped cells only from $8 \mathrm{kHz}$ on. This peculiar behavior reveals a certain conductivity of the unswitched MWCNTs. It is unlikely that the contribution of CNT charge transport normally to the axis may account for this decrease in impedance. Three alternative possibilities are currently under consideration: (i) a fraction of the CNTs is not aligned parallel to the LC director, but showing significant deviations; this fraction would foster more efficiently the conductivity of the mixture [29]; (ii) CNTs can contain ionic impurities that may diffuse into the LC upon sonication during the manufacturing process. Despite this, commercial CNTs are presumed to have low content of ionic impurities; (iii) ionic 

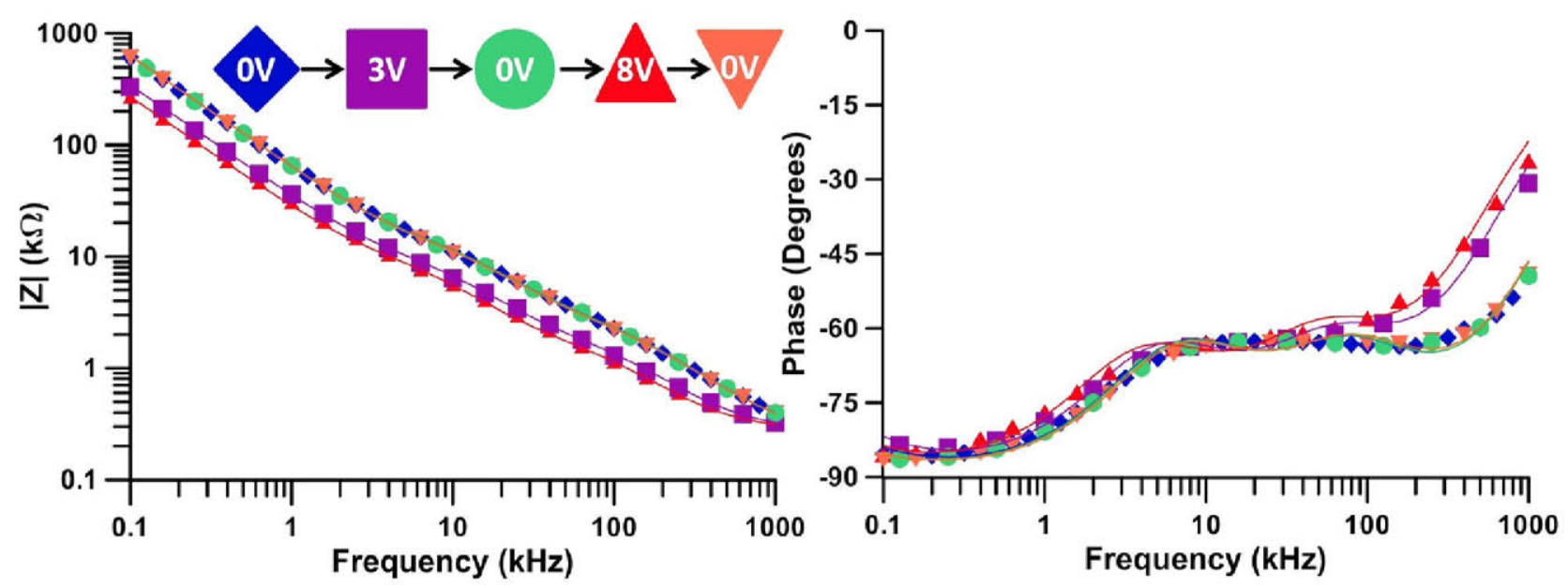

Figure 4. Bode plot of an undoped LC cell. Magnitude is shown on the left and phase on the right. Dots are experimental points while lines are calculated results of the EEC model. Inset: voltage sequence.
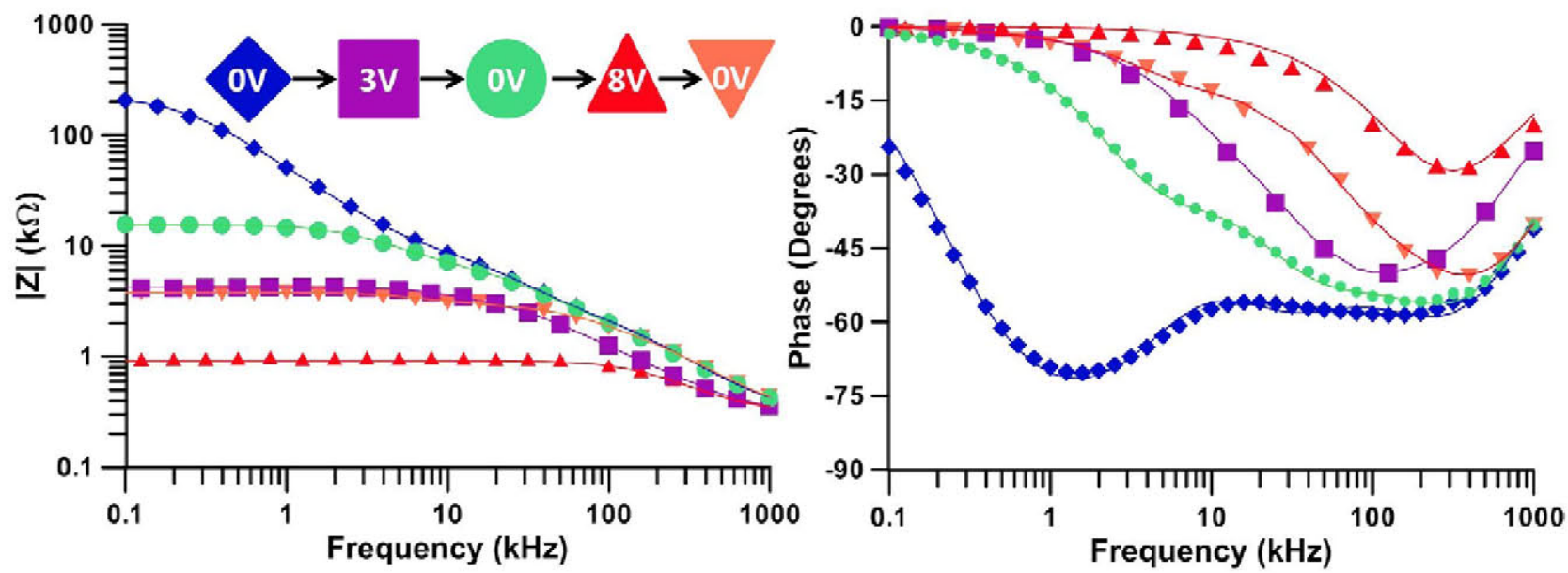

Figure 5. Bode plot of an MWCNT-doped LC cell. Symbols and features as in figure 4.

transport through the charge exchange mechanism is boosted by CNTs. The high dielectric constant of the CNTs attracts the impurity ions from the LC blend to the CNT surface [30].

The use of PEDOT:PSS as alignment layer may have some influence as well. As commented above, this layer is preferred to other traditional alignment layers like the aforementioned polyimides or PVA since these layers would mask low frequency phenomena. For example, the charge exchange mechanism commented above can be observed in the change experienced by the imaginary part of the dielectric constant of the doped cell. This effect results in a deviation of the phase at low frequencies at $0 \mathrm{~V}$ in figure 5 . The imaginary part of the permittivity increases at low frequencies when the MWCNTs are present in the PEDOT-LC system, increasing dielectric losses and reducing the impedance magnitude. This effect has been observed in other studies as well $[31,32]$. Thus, the change in the impedance seems the result of two combined effects: the electrical interaction of the alignment layer with the MWCNT, and the lack of orientation of a MWCNT fraction in the blend.

Once the driving voltage increases (figure 5), the capacitive behavior evolves towards a resistive behavior (an ideal resistor is a horizontal line in the Bode plot, since it is independent of frequency; its phase is zero). At $3 \mathrm{~V}_{\mathrm{p}}$ the resistor behavior prevails up to $10 \mathrm{kHz}$, while at $8 \mathrm{~V}_{\mathrm{p}}$ the resistor is seen over all but highest frequencies: the impedance magnitude is constant over nearly the whole frequency range tested while the phase is close to zero. The impedance magnitude at low frequency decreases almost two orders of magnitude at $3 \mathrm{~V}_{\mathrm{p}}$, and decreases further to a value lower than $1 \mathrm{k} \Omega$ at $8 \mathrm{~V}_{\mathrm{p}}$. This dramatic decrease in the impedance can only be explained by a reorientation of the MWCNTs that contributes to the charge transport through the LC cell.

The reorientation is barely reversible, as seen by the intermediate $0 \mathrm{~V}_{\mathrm{p}}$ measurements. Each measurement shows a certain recovery of the impedance relative to its previous biased run; yet the impedance is quite far from its original value. The irreversibility is independent of the time elapsed between successive runs, from a few seconds to several days. Optical inspection of the samples between crossed polarizers with no applied field shows permanent color changes in the active areas of the cell as compared to unswitched areas. These color changes derive from a modification of the optical phase delay, $\Delta$ :

$$
\Delta=\frac{2 \pi}{\lambda} \Delta n \cdot d
$$


Table 1. Undoped LC cell EEC components variation.

\begin{tabular}{llllllll}
\hline Driving sequence & $\mathrm{R}_{1}(\Omega)$ & $\mathrm{R}_{2}(\mathrm{k} \Omega)$ & $\mathrm{C}_{1}(\mathrm{nF})$ & $\mathrm{C}_{2}(\mathrm{nF})$ & $\mathrm{C}_{3}(\mathrm{nF})$ & $\mathrm{R}_{3}(\Omega)$ & $\mathrm{R}_{4}(\mathrm{M} \Omega)$ \\
\hline 0V & 254.8 & 7.36 & 2.15 & 0.73 & 1.77 & 2716 & 7.15 \\
3V & 283.5 & 5.05 & 4.14 & 1.44 & 3.18 & 1784 & 2.75 \\
0V after 3 V & 253.8 & 7.26 & 2.17 & 0.73 & 1.78 & 2694 & 9.29 \\
$8 \mathrm{~V}$ & 285.0 & 4.90 & 5.40 & 1.77 & 5.40 & 1661 & 3.12 \\
0V after 8 V & 253.2 & 6.97 & 2.23 & 0.73 & 1.78 & 2586 & 9.52 \\
\hline
\end{tabular}

Table 2. MWCNT-doped LC cell EEC components variation.

\begin{tabular}{llllllrr}
\hline Driving sequence & $\mathrm{R}_{1}(\Omega)$ & $\mathrm{R}_{2}(\mathrm{k} \Omega)$ & $\mathrm{C}_{1}(\mathrm{nF})$ & $\mathrm{C}_{2}(\mathrm{nF})^{\mathrm{a}}$ & $\mathrm{C}_{3}(\mathrm{nF})^{\mathrm{a}}$ & $\mathrm{R}_{3}(\Omega)^{\mathrm{a}}$ & $\mathrm{R}_{4}(\mathrm{k} \Omega)^{\mathrm{a}}$ \\
\hline 0 V & 312.9 & 4.66 & 2.12 & 0.80 & 2.30 & 2006 & 218.62 \\
$3 \mathrm{~V}$ & 311.4 & 2.00 & 2.06 & 2.15 & 4.37 & 575 & 1.94 \\
0V after 3 V & 309.5 & 4.16 & 1.58 & 0.91 & 3.40 & 1443 & 11.00 \\
$8 \mathrm{~V}$ & 316.5 & 0.61 & 1.42 & - & - & - & 1328 \\
0V after 8 V & 291.4 & 1.88 & 1.51 & 0.99 & 8.28 & 1.64 \\
\hline
\end{tabular}

a The values of these components could not be determined at $8 \mathrm{~V}_{\mathrm{p}}$ because their effect in impedance is negligible compared to $\mathrm{R}_{1}$, $\mathrm{R}_{2}$ and $\mathrm{C}_{1}$; consequently their values are undefined.

which has to be attributed to a modification of the birefringence $\Delta n$, since the thickness $d$ is constant. This confirms that some optically anisotropic component of the LC blend has not reversibly relaxed to its original position after removing the electric field, and it should be considered as an optical consequence of the MWCNT orientation into the blend.

This lack of reversibility has been reported by other authors [4]. It is attributed to the existence of a swarm of LC molecules binding the CNTs, aligned parallel to their long axes. Consequently, pseudo-nematic domains are formed around every CNT; this LC fraction remains aligned with the CNT without relaxing to its original position by the elastic restoring forces.

An indication on the actual existence of these domains is the higher order parameter observed in the CNT-LC mixture as compared to the pure $\mathrm{LC}$; moreover, dielectric anisotropy has been detected [32] in the mixture above the LC isotropic transition. This anisotropy implies a certain long-range order that cannot be caused by the LC alone.

Some attempts have been made in our laboratory to verify this interesting result by optical measurements. Preliminary results obtained in a Mach-Zehnder interferometer setup seem to confirm this effect: applying voltage above threshold in isotropic state, phase delays are detected in doped cells. These delays should arise from the reorientation of the LC pseudonematic fraction attached to the CNTs. Confirmation and quantification of these results are currently under study.

Interestingly enough, it has been recently found [33, 34] that CNT mixtures in LCs with negative dielectric anisotropy are reversible upon switching, at least for low CNT concentrations.

\section{EEC study}

Fitting these Bode plots to EECs is not simple, since the samples show different slope variations of the magnitude and phase in the frequency range under study. A number of electric components is required for the EEC to follow such a complex response. As mentioned above, a comprehensive circuit originally proposed for LC nematic displays (figure 2) has been tested also for doped cells. The study [23] gives an approximation of the EEC components magnitudes for nematic LCs in homogeneous (i.e. planar) alignment. The values of our retrieved components (table 1) at the unbiased state are in concordance with the ones of that study, given that our samples are narrower and our alignment layer is more conductive. Once the driving voltage is applied to the sample, the undoped LC cell EEC components present a logical variation: LC reorientation to homeotropic (perpendicular) director increases the dielectric permittivity experienced by the applied electric field. Consequently, the capacitor values increase (as capacitance depends directly on the dielectric permittivity value) and the resistor values decrease (because the energy dissipation is lower in homeotropic than in planar alignment). The undoped cell presents reversible behavior, as every component recovers its original value once the driving signal is switched off.

The MWCNT-doped LC cells present many divergences respect to the undoped $\mathrm{LC}$ cells. Whether the same EEC is applicable to doped cells or not can be assessed by checking the evolution of every component with the voltage sequence.

The changes in the component values of MWCNT-doped LC cells with voltage (table 2) are within one order of magnitude (except for R4), yet it is possible to observe the new features introduced by the MWCNTs. The presence of CNTs in the mixture increases the conductivity of the dielectric blend (i.e. decreases the R4 value) in one order of magnitude but its initial planar alignment does not modify the remaining parameters.

Other effects occur when driving voltage is applied to the cells:

$\mathrm{R} 1$ is virtually constant with the driving voltage. The adjusted value fits well with the geometry of ITO-electrodes of the cell. The ITO resistance is $100 \Omega / \square$, and the electrodes are two crossed rectangles with an aspect ratio of $2: 1$, overlapping in a square. Thus, $R 1$ can be assumed as the series of both non-overlapping parts of electrodes (two squares, i.e. $100 \Omega$ 
each) plus the effect of the overlapping part, with a total estimated resistance of around $300 \Omega$.

$\mathrm{R} 2$ decreases (as in the undoped LC cell) when the driving voltage is applied; $\mathrm{C} 1$ decreases as well. Since MWCNT charge transport is much more efficient in the longitudinal rather than in the transversal axis, MWCNT reorientation to the perpendicular direction and possible penetration into the alignment layers can establish electric paths inside the alignment layers, increasing the current in the direction normal to the plates and the conductivity of the substrate layers, decreasing the resistance associated to these layers. This alters the charge distribution in the PEDOT : PSS/LC interface, which determines the double layer capacitance related to this substrate (C1). R2 and $\mathrm{C} 1$ do not recover their original value once the driving voltage is suppressed; consequently MWCNTs, or at least a fraction of them, do not come back to the original position [4] and a number of electrical paths become permanent.

$\mathrm{C} 2$ and $\mathrm{C} 3$ increase their values too, due to the increase of dielectric permittivity when MWCNT doped LC cells are excited. As mentioned above, strong anchoring between the LC molecules and CNTs [32] stabilizes pseudonematic domains about every CNT, even in the presence of electric field or in isotropic state. The restoring elastic forces from the cell surface anchoring are not strong enough to bring the MWCNTs (or a fraction of them) to the relaxed planar orientation once the field is removed. Regarding R3, the evolution shows a decrease when voltage is applied, quite similar in doped and undoped cells, with lower values in the first case. This resistance is linked to the LC material, specifically with the dipolar displacement, which is favored by the applied voltage and the CNT presence. Moreover, R3 does not return to its former value when voltage is removed. This could be explained again assuming a fraction of CL molecules associated to the CNTs that does not relax to their original orientation, thus modifying the dipolar displacement.

$\mathrm{R} 4$ is the EEC element having the most different behavior in comparison to its value and evolution in undoped LC cells. This element, as standing for the dielectric conductivity, could confirm the MWCNT reorientation along with the reorientation of LC molecules and the existence of electrical paths between the electrodes. Increasing the driving voltage, R4 decreases (figure 6). An interesting phenomenon is observed at $8 \mathrm{~V}_{\mathrm{p}}$. The conductivity of the dispersed CNTs is so high that estimating the value of $\mathrm{R} 4$ becomes impossible. In a parallel configuration of electrical components, the lower impedance is dominating. As R4 becomes very low, the effect of the remaining components connected in parallel $(\mathrm{C} 2, \mathrm{C} 3$ and $\mathrm{R} 3)$ is masked. The final result is that the equivalent circuit shows up two resistors in series (R1 and R4), while $\mathrm{R} 2$ remains in parallel with $\mathrm{C} 2$. Regarding the fitting algorithm, the two series resistors are equivalent to one resistor, being impossible to split both components. Besides, the series value is similar to previous $\mathrm{R} 1$ values. This indicates that the value of $\mathrm{R} 4$ has to be within the error margins of R1 estimation. An upper limit for R4 in this context would be $10 \Omega$.

Considering the above arguments, measurements at $8 \mathrm{Vp}$ could be justified with a simplified model of the original EEC formed only by R1, R2 and C1 (figure 6, inset). R4 should

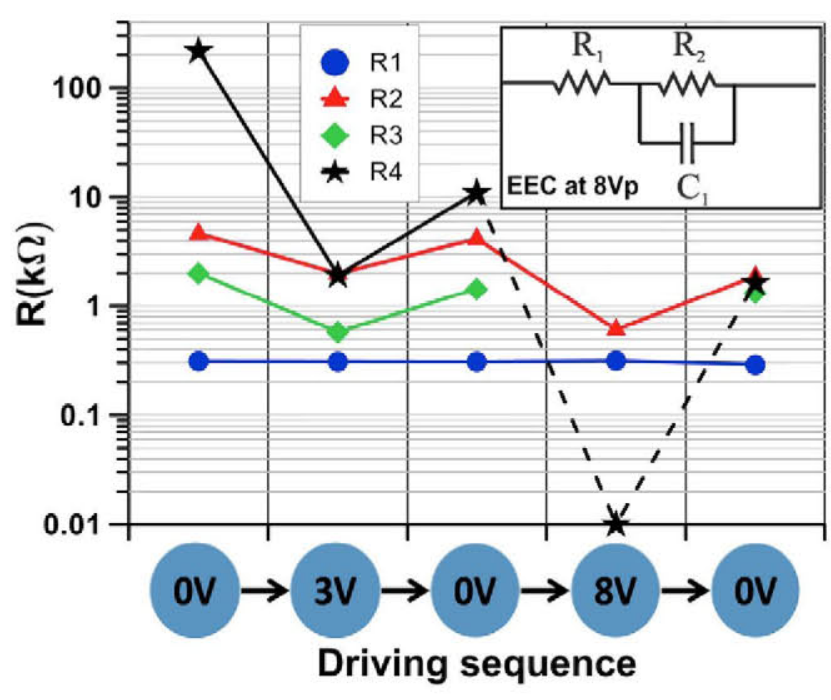

Figure 6. Evolution of resistive components of the EEC. Inset: reduced circuit at $8 \mathrm{~V}_{\mathrm{p}}$. R4 @8V is an upper estimation. R3 @8V is masked by R4 and cannot be estimated.

appear in series with that set, but as deduced above, its negligible value is hidden in the $\mathrm{R} 1$ fit. When the saturation voltage is removed (last measurement at $0 \mathrm{~V}_{\mathrm{p}}$ ), the description of impedance behavior requires again the use of the original EEC. Nevertheless, the results show a non-reversible performance: R4 does not recover its original initial value once the driving voltage has been removed. This confirms the existence of short-circuits or low impedance electrical paths between the electrodes. The impedance behavior is then dominated by the electrodes resistivity.

A rough estimation of the fraction of switched MWCNTs not coming back to the relaxed planar state can be done under several assumptions. It will be assumed that the contribution to cell conductivity of any CNT element is the same. CNTs are quite rigid, no folding of the nanotubes is expected; therefore this assumption is equivalent to assume that all non-relaxed CNTs remain in similar orientations, whether perpendicular or tilted. The ohmic resistance in either case would be,

$$
\frac{1}{R_{T}}=\sum_{i=1}^{N} \frac{1}{R_{i}}=\frac{N}{R_{i}}
$$

where all $R_{i}$ are assumed to be the same (they should be quite close to each other, actually), and $R_{T}$ is the resistance of the parallel. Comparing the values at $8 \mathrm{~V}$ and $0 \mathrm{~V}$ after $8 \mathrm{~V}(<10$ $\Omega$ and $1.64 \mathrm{k} \Omega$ respectively), it is easy to estimate that about $0.60 \%$ of the CNTs remaining fully switched would give such a resistance. The same calculation can be done at $3 \mathrm{~V}_{\mathrm{p}}$. In this case, the orientation adopted by the MWCNTs would be a tilted intermediate position between planar and homeotropic, but the derivation is the same. Applying the same formula, the estimation of CNTs remaining partially switched (tilted) is about $17.6 \%$. However, if the conductivity at $0 \mathrm{~V}$ after $3 \mathrm{~V}$ is attributed to a fraction of fully switched MWCNTs, such a fraction would be just $0.091 \%$. This remarkable disparity is explained by the large difference between the conductivities of fully and partially switched CNTs. Nevertheless, a more 
precise estimation of the switched fractions and the switching mechanism is presently under study.

\section{Conclusions}

The electrical behavior of MWCNT-doped LC cells has been studied by impedance measurements and modeled with an electrical equivalent circuit. Several internal effects have been explained using these results. The same EEC circuit proposed for undoped LC cells can be used for modeling doped cells, but the evolution with frequency and driving voltage is significantly different. In undoped cells, a predominant capacitive behavior from $100 \mathrm{~Hz}$ to $1 \mathrm{MHz}$ has been confirmed, with a capacity value depending on the applied voltage, as the LC molecules orient with electric field. Doping with MWCNTs generates changes even with no applied voltage. A decrease in the capacity in the same frequency range and a predominant resistive behavior at low frequencies have been related to the combined result of two effects: the misalignment of some MWCNTs, and their electrical interaction with the PEDOT : PSS alignment layer. The optical differences between the doped and undoped devices also confirm that some nematic domains are formed around the MWCNTs due to their anchoring forces on the LC molecules, an effect seeing also by the decrease of R3 value in the EEC. EEC evolution shows that some of these domains remain oriented after voltage switching (by the decrease of R3 value with voltage). At higher voltages, MWCNTs create electrical paths between contacts, as seen by the huge drop of R4, that leads even to a short in the EEC part related to the dielectric blend conductivity. The effect is scarcely reversible, as seen by the EEC components not recovering their original values when the exciting voltage is suppressed. Summing up, this study reveals that MWCNTs interact strongly with the LC molecules and could alter the capacitive properties of LC cells, even turning them into an irreversibly conductive state above a threshold voltage. This kind of structures could be used as permanent memories or components for protection circuits.

\section{Acknowledgments}

This work has been supported by Program RETOS of the Spanish Ministerio de Economía y Competitividad, project TEC2013-47342-C2, the R\&D Program SINFOTON S2013/ MIT-2790 of the Comunidad de Madrid and the European COST Action IC1208.

\section{References}

[1] Blanc C, Coursault D and Lacaze E 2013 Liq. Cryst. Rev. 1 83-109

[2] Bisoyi H and Kumar S 2011 Chem. Soc. Rev. 40 306-19
[3] Sridevi S, Prasad S K, Nair G and D'Britto V 2010 Appl. Phys. Lett. 97151913

[4] Basu R and Iannacchione G S 2009 Appl. Phys. Lett. 95173113

[5] Li X, Yang C, Wang Q, Jia D, Hu L, Peng Z and Xuan L 2013 Opt. Commun. $286224-7$

[6] Singh U B, Dhar R, Dabrowski R and Pandey M B 2013 Liq. Cryst. $40774-82$

[7] Ha Y, Kim H, Park H and Seo D 2012 Opt. Express 20 6448-55

[8] Dierking I, Scalia G and Morales P 2005 J. Appl. Phys. 97044309

[9] García-García A, Vergaz R, Algorri J F, Quintana X and Otón J M 2015 Beilstein J. Nanotechnol. 6 396-403

[10] Dolgov L, Kovalchuk O, Lebovka N, Tomylko S and Yaroshchuk O 2010 Liquid Crystal Dispersions of Carbon Nanotubes: Dielectric, Electro-Optical and Structural Peculiarities in Carbon Nanotubes ed J M Marulanda (Rijeka, Croatia: InTech)

[11] Patrick D and Lynch M 2002 Nano Lett. 2 1197-201

[12] Brochard F and De Gennes P 1970 J. Phys. 31 691-708

[13] Burylov S and Raikher Y L 1990 Phys. Lett. A 149 279-83

[14] van der Schoot P, Popa Nita V and Kralj S 2008 J. Phys. Chem. B 112 4512-8

[15] Rahman M and Lee W 2009 J. Phys. D: Appl. Phys. 42063001

[16] Schymura S and Scalia G 2013 Phil. Trans. R. Soc. A 37120120261

[17] Dierking I, Casson K and Hampson R 2008 Japan. J. Appl. Phys. 47 6390-3

[18] Dierking I and San E 2005 Appl. Phys. Lett. 87233507

[19] Dierking I, Scalia G, Morales P and LeClere D 2004 Adv. Mater: 16 865-9

[20] Oliva-Avilés A, Avilés F, Sosa V, Oliva A and Gamboa F 2012 Nanotechnology 23465710

[21] Baughman R H, Zakhidov A A and de Heer W A 2002 Science 297 787-92

[22] Volpati D, Massey M, Johnson D, Kotsialos A, Qaiser F, Pearson C, Coleman K, Tiburzi G, Zeze D and Petty M 2015 J. Appl. Phys. 117125303

[23] Costa M R, Altafim R A C and Mammana A P 2006 IEEE Trans. Dielectr. Electr. Insul. 13 204-10

[24] Torres Zafra J C, Perez Garcilopez I, Urruchi del Pozo V, Sanchez Pena J M and Marcos Lucas C 2011 Opt. Eng. 50 081206

[25] Pena J M S, Perez I, Urruchi V, Torres J C and Otón J M 2008 Opto-Electron. Rev. 16 189-93

[26] Macdonald J R 1987 J. Electroanal. Chem. 223 25-50

[27] de Paula J, Santoro P, Zola R, Lenzi E, Evangelista L, Ciuchi F, Mazzulla A and Scaramuzza N 2012 Phys. Rev. E 86051705

[28] Basu R and Iannacchione G S 2008 Appl. Phys. Lett. 93183105

[29] Behnam A, Guo J and Ural A 2007 J. Appl. Phys. 102044313

[30] Baik I, Jeon S Y, Lee S H, Park K A, Jeong S H, An K H and Lee Y H 2005 Appl. Phys. Lett. 87263110

[31] Perkowski P, Łada D, Ogrodnik K, Rutkowska J, Piecek W and Raszewski Z 2008 Opto-Electron. Rev. 16 271-6

[32] Basu R and Iannacchione G S 2010 Phys. Rev. E. 81051705

[33] Dolgov L, Yaroshchuk O, Tomylko S and Lebovka N 2012 Condens. Matter Phys. $\mathbf{1 5} 33401$

[34] Kalakonda P, Basu R, Nemitz I, Rosenblatt C and Iannacchione G 2014 J. Chem. Phys. 140104908 